\title{
Fusobacterium nucleatum in gastroenterological cancer: Evaluation of measurement methods using quantitative polymerase chain reaction and a literature review
}

\author{
KENSUKE YAMAMURA ${ }^{1}$, YOSHIFUMI BABA ${ }^{1}$, KEISUKE MIYAKE ${ }^{1}$, KENICHI NAKAMURA ${ }^{1}$, \\ HIRONOBU SHIGAKI ${ }^{1}$, KOSUKE MIMA ${ }^{1}$, JUNJI KURASHIGE ${ }^{1}$, TAKATSUGU ISHIMOTO ${ }^{1}$, MASAAKI IWATSUKI ${ }^{1}$, \\ YASUO SAKAMOTO $^{1}$, YOICHI YAMASHITA ${ }^{1}$, NAOYA YOSHIDA ${ }^{1}$, MASAYUKI WATANABE $^{2}$ and HIDEO BABA ${ }^{1}$ \\ ${ }^{1}$ Department of Gastroenterological Surgery, Graduate School of Medical Sciences, Kumamoto University, \\ Kumamoto 860-8556; ${ }^{2}$ Department of Gastroenterological Surgery, Cancer Institute Hospital, \\ Japanese Foundation for Cancer Research, Tokyo 135-8550, Japan
}

Received June 17, 2016; Accepted February 13, 2017

DOI: $10.3892 / \mathrm{ol} .2017 .7001$

\begin{abstract}
The human microbiome Fusobacterium nucleatum, which primarily inhabits the oral cavity, causes periodontal disease and has also been implicated in the development of colorectal cancer. However, whether F. nucleatum is present in other gastroenterological cancer tissues remains to be elucidated. The present study evaluated whether quantitative polymerase chain reaction (qPCR) assays were able to detect $F$. nucleatum DNA and measure the quantity of $F$. nucleatum DNA in esophageal, gastric, pancreatic and liver cancer tissues. The accuracy of the qPCR assay was determined from a calibration curve using DNA extracted from cells from the oral cavity. Formalin-fixed paraffin-embedded (FFPE) tumor tissues from 20 patients with gastroenterological [esophageal (squamous cell carcinoma), gastric, colorectal, pancreatic and liver] cancer and 20 matched normal tissues were evaluated for $F$. nucleatum DNA content. The cycle threshold values in the qPCR assay for $F$. nucleatum and solute carrier organic anion transporter family member 2A1 (reference sample) decreased linearly with the quantity of input DNA $\left(r^{2}>0.99\right)$. The $F$. nucleatum detection rate in esophageal, gastric and colorectal cancer tissues were $20 \%(4 / 20), 10 \%(2 / 20)$ and $45 \%$ (9/20), respectively. F. nucleatum was not detected in liver and pancreatic cancer tissues. The qPCR results from the frozen and FFPE tissues were consistent. Notably, F. nucleatum was detected at a higher level in superficial areas compared with the invasive areas. F. nucleatum in esophageal, gastric and colorectal cancer tissues was evaluated by qPCR using FFPE
\end{abstract}

Correspondence to: Professor Hideo Baba, Department of Gastroenterological Surgery, Graduate School of Medical Sciences, Kumamoto University, 1-1-1 Honjo, Kumamoto 860-8556, Japan E-mail: hdobaba@kumamoto-u.ac.jp

Key words: microbiome, Fusobacterium, gastroenterological cancer, esophageal cancer, reverse transcription-quantitative polymerase chain reaction tissues. F. nucleatum may be involved in the development of esophageal, gastric and colorectal cancer.

\section{Introduction}

As a developing research area, the microbiome has been the focus of multiple studies in previous years. The non-spore-forming, anaerobic gram-negative bacterium Fusobacterium nucleatum is part of the normal flora of the human oral cavity and gut mucosa, but is an established opportunistic pathogen in periodontal diseases (1-4) and several inflammatory diseases, including inflammatory bowel disease (5-8), liver abscesses $(9,10)$ and chorioamnionitis (11). Two previous studies have reported an overabundance of $F$. nucleatum in colorectal cancer tissues compared with adjacent normal tissues $(12,13)$. Following this, a previous study demonstrated that $F$. nucleatum activates the E cadherin $/ \beta$-catenin signaling pathway via FadA adhesion, promoting colorectal cancer growth (14). Fusobacterium subspecies (spp.), including $F$. nucleatum, are also present at increased levels in human colorectal, pancreatic and other types of cancer (12,13,15-20). To the best of our knowledge, there are only five previous studies reporting the presence of Fusobacterium spp. in colorectal and pancreatic cancer tissues and there are no published studies that associate Fusobacterium spp. with esophageal, gastric, hepatocellular and other gastroenterological cancer (Table I) $(15,16,19,20,21)$.

Elevated levels of F. nucleatum DNA in colorectal cancer tissue are associated with certain molecules and cell functions, including microsatellite instability, the $\mathrm{CpG}$ island methylator phenotype and hMLH1 (15), and are also associated with a lower density of $\mathrm{T}$ cells (16). A number of previous studies have associated high levels of $F$. nucleatum DNA content with poor patient prognosis $(17,18)$, however, other previous studies have reported that there is no association between the quantity of $F$. nucleatum DNA and patient survival rate $(12,19)$. In one previous study, the DNA status of Fusobacterium spp. in pancreatic cancer tissue was independently associated with the poor prognosis of patients (20). 
However, whether $F$. nucleatum is present in other types of gastroenterological cancer, including esophageal, gastric or liver cancer, has yet to be investigated.

In the present study, quantitative polymerase chain reaction (qPCR) method was evaluated to determine if it was able to detect the quantity of $F$. nucleatum DNA from an oral cavity. Subsequently, a qPCR assay was also used to analyze whether it similarly detects the existence of Fusobacterium in formalin-fixed paraffin-embedded (FFPE) tissues and frozen tissues. Finally, the quantity of $F$. nucleatum DNA in 20 paraffin-embedded digestive cancer specimens and 20 matched normal specimens was evaluated.

\section{Materials and methods}

Tissue samples. The test specimens were 20 FFPE tissue samples of esophageal (squamous cell carcinoma), gastric, colorectal, liver and pancreatic cancer, and 20 normal matched paraffin embedded specimens. All specimens were obtained by surgical resection at Kumamoto University Hospital (Kumamoto, Japan). The sampled patients were not administered preoperative treatment. A single pathologist, who was blind to the clinical and molecular data of the patients, evaluated hematoxylin-eosin-stained tissue sections of each cancer case and recorded the pathological features. Tumor staging was conducted as described in the Cancer Staging Manual (7th edition) published by the American Joint Committee on Cancer (22). Written informed consent was obtained from each patient and the present study was approved by the Institutional Review Board of Kumamoto University Hospital (Approval no. 1272).

DNA extraction and qPCR for F. nucleatum DNA content. Genomic DNA in the oral cavity was obtained using a cotton swab. The patients were not allowed to eat or drink 30 min prior to sample collection and the cotton swap was scraped against the inside of each cheek 5-6 times. The collected swab was air-dried for $>2 \mathrm{~h}$. The genomic DNA from the oral cavity was extracted using QIAamp DNA Mini kit (Qiagen GmbH, Hilden, Germany). Genomic DNA from the FFPE tissues and from the frozen gastroenterological cancer tissues was extracted using the QIAamp DNA FFPE Tissue kit (Qiagen $\mathrm{GmbH}$ ) and the QIAamp DNA Mini kit (Qiagen $\mathrm{GmbH}$ ), respectively. The $n u s G$ gene of $F$. nucleatum and the reference human gene solute carrier organic anion transporter family member 2A1 (SLCO2A1) were amplified using custom-made TaqMan primer/probe sets (Applied Biosystems; Thermo Fisher Scientific, Inc., Waltham, MA, USA) as previously described (18). The primer and probe sequences used for the custom TaqMan Gene Expression assay were as follows: F. nucleatum forward primer, 5'-TGG TGTCATTCTTCCAAAAATATCA-3'; F. nucleatum reverse primer, 5'-AGATCA AGA AGGACA AGTTGCTGA A-3'; $F$. nucleatum FAM probe, 5'-ACTTTAACTCTACCATGT TCA-3'; SLCO2A1 forward primer, 5'-ATCCCCAAAGCA CCTGGTTT-3'; SLCO2A1 reverse primer, 5'-AGAGGCCAA GATAGTCCTGGTAA-3'; SLCO2A1 VIC probe, 5'-CCATCC ATGTCCTCATCTC-3'. The PCR mix consisted of 1X TaqMan Environmental Master Mix 2.0 (Applied Biosystems; Thermo Fisher Scientific, Inc.), $0.5 \mathrm{pmol}$ forward and reverse primer, 0.1 pmol probe, nuclease-free water (Invitrogen; Thermo Fisher
Scientific, Inc.) and $12.5 \mathrm{ng}$ genomic DNA in a total volume of $10 \mu 1$. Assays were performed in a 384-well optical PCR plate. The DNA was amplified and detected with the LightCycler 480 Instrument II (Roche Diagnostics, Basel, Switzerland) under the following reaction conditions: Initial denaturation at $95^{\circ} \mathrm{C}$ for $10 \mathrm{~min}, 15 \mathrm{sec}$ at $95^{\circ} \mathrm{C}$ and $60 \mathrm{sec}$ at $60^{\circ} \mathrm{C}$. The quantity of $F$. nucleatum DNA in each tissue was normalized relative to SLCO2A1 using the $2^{-\Delta \Delta \mathrm{Cq}}$ method (where $\Delta \mathrm{Cq}$ is the mean $\mathrm{Cq}$ of F. nucleatum minus the mean Cq of SLCO2A1) $(16,23)$. All $\mathrm{RT}-\mathrm{qPCR}$ reactions were performed in triplicate.

Statistical analysis. All statistical analyses were performed by the JMP program, version 10 (SAS Institute, Inc., Cary, NC, USA). All P-values were 2-sided. The mean quantity of $F$. nucleatum DNA was compared with paired Student's $t$-tests for variables with two categories. $\mathrm{P}<0.05$ was considered to indicate a statistically significant difference.

\section{Results}

Literature review. An online search of MEDLINE (PubMed) was performed for all articles published in the English language. The following Medical Subject Headings terms were used in combination: 'Fusobacterium esophageal cancer', 'Fusobacterium gastric cancer', 'Fusobacterium colorectal cancer', 'Fusobacterium hepatocellular carcinoma', and 'Fusobacterium pancreatic cancer'. The latest search was performed on December 2015. Among them, five studies which had detection rates of Fusobacterium spp. in cancer tissues were identified. In total, four previous studies have reported detectable levels of $F$. nucleatum in colorectal cancer tissues $(15,16,19,21)$. The F. nucleatum detection rate was $13-82 \%$ in colorectal tumor tissue and 3.4-81\% in adjacent normal tissue (Table I). A single previous study detected $F$. nucleatum in pancreatic cancer (the detection rate was $8.8 \%$ in tumor tissue and $28 \%$ in adjacent normal tissue) (20). However, the expression status of Fusobacterium DNA in esophageal, gastric and liver cancer remains to be elucidated.

Validation of $q P C R$ for $F$. nucleatum. A cheek swab from a healthy researcher (Dr Kensuke Yamamura, Department of Gastroenterology, Kumamoto University, Kumamoto, Japan) was submitted for genomic DNA determination of the oral cavity. F. nucleatum and SLCO2A1 in the oral cavity were evaluated using qPCR in a 2-fold dilution series $(5,10,12.5$, 20 and $40 \mathrm{ng}$ ). The assays were quantified using the coefficient of determination $\left(r^{2}\right)$ between 5 and $40 \mathrm{ng}$. In the qPCR assays of oral F. nucleatum and SLCO2A1, the cycle threshold $(\mathrm{Cq})$ values linearly decreased with the quantity of input DNA (on a linear-log scale, $r^{2}>0.99$; Fig. 1). These results demonstrated that qPCR has the ability to quantify $F$. nucleatum DNA in the oral cavity.

qPCR of F. nucleatum in frozen tissue and FFPE. F.nucleatum DNA in FFPE and frozen tissues of 10 esophageal squamous cell carcinoma (ESCC) cases were investigated. In the 5 tissues that were positive for F. nucleatum, the organism was also detected in the matched FFPE tissues. Similarly, in the 5 Fusobacterium-negative ESCC cases, F. nucleatum was not detected in the matched FFPE tissue (Table II). Therefore, the 
Table I. Detection rates of Fusobacterium spp. in gastroenterological cancer tissues from previous studies.

\begin{tabular}{|c|c|c|c|c|c|c|c|}
\hline \multirow[b]{2}{*}{ Authors } & \multirow{2}{*}{$\begin{array}{l}\text { Type of } \\
\text { cancer }\end{array}$} & \multirow{2}{*}{$\begin{array}{r}\text { No. of } \\
\text { cases }\end{array}$} & \multirow{2}{*}{$\begin{array}{l}\text { Tissue } \\
\text { fixation }\end{array}$} & \multirow{2}{*}{$\begin{array}{l}\text { Bacterial } \\
\text { strain }\end{array}$} & \multicolumn{2}{|c|}{ Fusobacterium detection rate, $\%$} & \multirow[b]{2}{*}{ (Refs.) } \\
\hline & & & & & Tumor tissue & Normal tissue & \\
\hline Tahara et al, 2014 & $\begin{array}{l}\text { Colorectal } \\
\text { cancer }\end{array}$ & 149 & $\begin{array}{l}\text { Frozen } \\
\text { tissue }\end{array}$ & F. nucleatum & $52.3(78 / 149)$ & $30.3(27 / 89)$ & $(15)$ \\
\hline Mima et al, 2015 & $\begin{array}{l}\text { Colorectal } \\
\text { cancer }\end{array}$ & 598 & FFPE & F. nucleatum & $13(76 / 598)$ & $3.4(19 / 558)$ & (16) \\
\hline Ito et al, 2015 & $\begin{array}{l}\text { Colorectal } \\
\text { cancer }\end{array}$ & 511 & FFPE & F.nucleatum & $56(286 / 511)$ & - & (19) \\
\hline Mitsuhashi et al, 2015 & $\begin{array}{l}\text { Pancreatic } \\
\text { cancer }\end{array}$ & 283 & FFPE & $\begin{array}{l}\text { Fusobacterium } \\
\text { species }\end{array}$ & $8.8(25 / 283)$ & $28(7 / 25)$ & $(20)$ \\
\hline Viljoen et al, 2015 & $\begin{array}{l}\text { Colorectal } \\
\text { cancer }\end{array}$ & 71 & FFPE & $\begin{array}{l}\text { F. nucleatum spp. } \\
\text { polymorphum }\end{array}$ & $82(58 / 71)$ & $81(48 / 59)$ & $(21)$ \\
\hline
\end{tabular}

spp., subspecies; FFPE, formalin-fixed paraffin-embedded tissues; F., Fusobacterium.

Table II. Consistency of quantitative polymerase chain reaction detection of Fusobacterium nucleatum in tumor FFPEs and frozen tissues of esophageal cancer.

\begin{tabular}{lccccccccccc}
\hline Variable & Case 1 & Case 2 & Case 3 & Case 4 & Case 5 & Case 6 & Case 7 & Case 8 & Case 9 & Case 10 \\
\hline FFPE & - & - & - & - & - & + & + & + & + \\
Frozen tissue & - & - & - & - & - & + & + & + & + & + \\
Concordance & Yes & Yes & Yes & Yes & Yes & Yes & Yes & Yes & Yes & Yes \\
\hline
\end{tabular}

FFPE, formalin-fixed paraffin-embedded tissues.

Table III. Quantitative polymerase chain reaction results of Fusobacterium nucleatum in gastroenterological cancer and adjacent normal tissues.

\begin{tabular}{lccc}
\hline & \multicolumn{3}{c}{$\begin{array}{c}\text { Fusobacterium detection } \\
\text { rate, } \%\end{array}$} \\
\cline { 2 - 4 } & $\begin{array}{c}\text { Tumor } \\
\text { tissue }\end{array}$ & $\begin{array}{c}\text { Normal } \\
\text { tissue }\end{array}$ & $\begin{array}{c}\text { Tumor and } \\
\text { normal tissues }\end{array}$ \\
\hline Esophageal cancer & $20(4 / 20)$ & $5(1 / 20)$ & 0 \\
Gastric cancer & $10(2 / 20)$ & 0 & 0 \\
Colorectal cancer & $45(9 / 20)$ & $40(8 / 20)$ & $25(5 / 20)$ \\
Liver cancer & 0 & 0 & 0 \\
Pancreatic cancer & 0 & 0 & 0 \\
\hline
\end{tabular}

qPCR results were consistent between the frozen tissues and FFPE tissues. These results suggested that F. nucleatum may be accurately assayed in FFPE tissues.

F. nucleatum in gastroenterological cancer tissue. 20 FFPE tumors and their adjacent non-tumorous tissues in each cancer were analyzed using qPCR assays. F. nucleatum was detected in $4(20 \%)$ cases of esophageal cancer, $2(10 \%)$ cases of gastric

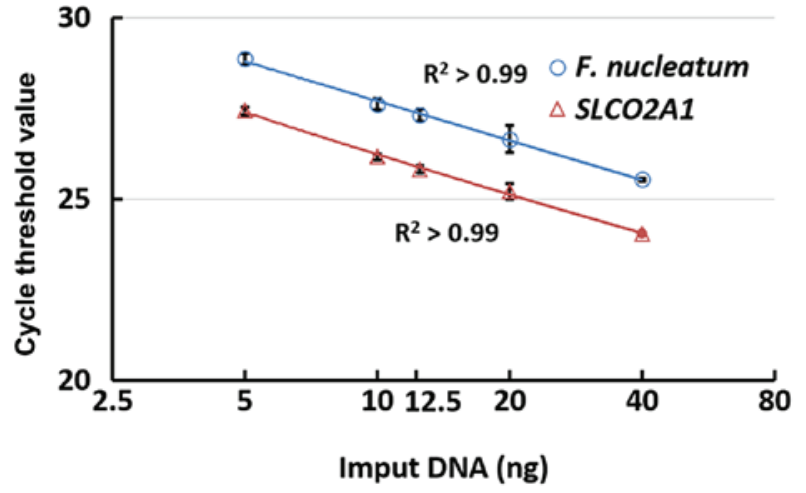

Figure 1. Analysis of linearity in quantitative polymerase chain reaction assay of $F$. nucleatum from the oral cavity. Levels of $F$. nucleatum and the human reference gene (SLCO2A1) from the oral cavity obtained by oral swab, demonstrated in a 2-fold dilution series (5, 10, 12.5, 20 and $40 \mathrm{ng}$ ). Data points and error bars denote the mean \pm standard deviation of the cycle thresholds, respectively. Results were performed with triplicate runs in three separate experiments. F., Fusobacterium; $\mathrm{R}^{2}$, coefficients of determination; SLCO2A1, solute carrier organic anion transporter family member $2 \mathrm{~A} 1$.

cancer and 9 (45\%) cases of colorectal cancer (Fig. 2; Table III). In esophageal and colorectal cancer, F. nucleatum was also detected in adjacent non-tumor tissue, whereas $F$. nucleatum was not detected in the liver and pancreatic cancer tissues 

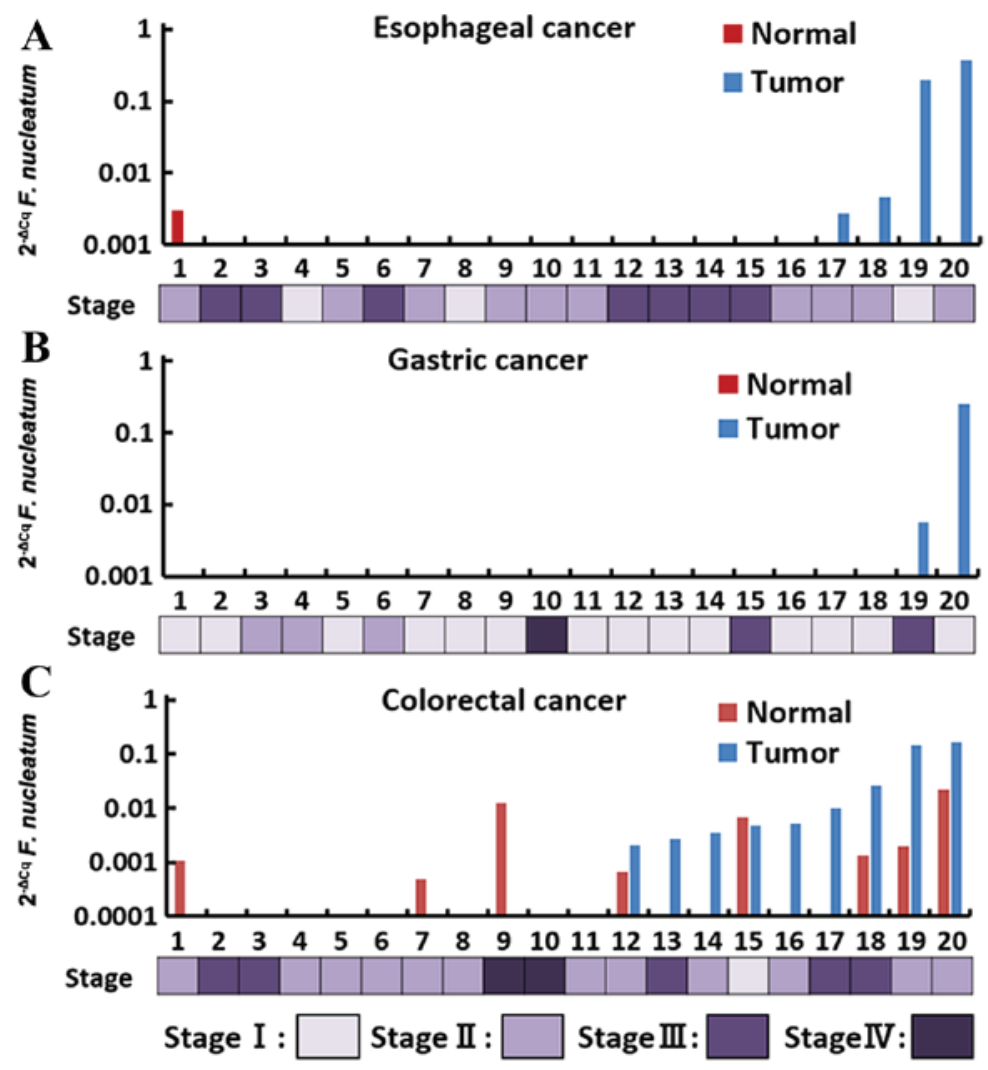

Figure 2. Relative quantity of $F$. nucleatum DNA in gastroenterological cancer. Quantitative polymerase chain reaction assays of $F$. nucleatum in the tumor and adjacent non-tumor tissues of 20 patients with (A) esophageal cancer, (B) gastric cancer and (C) colorectal cancer. The cancer stage of each tumor is also presented. F., Fusobacterium.
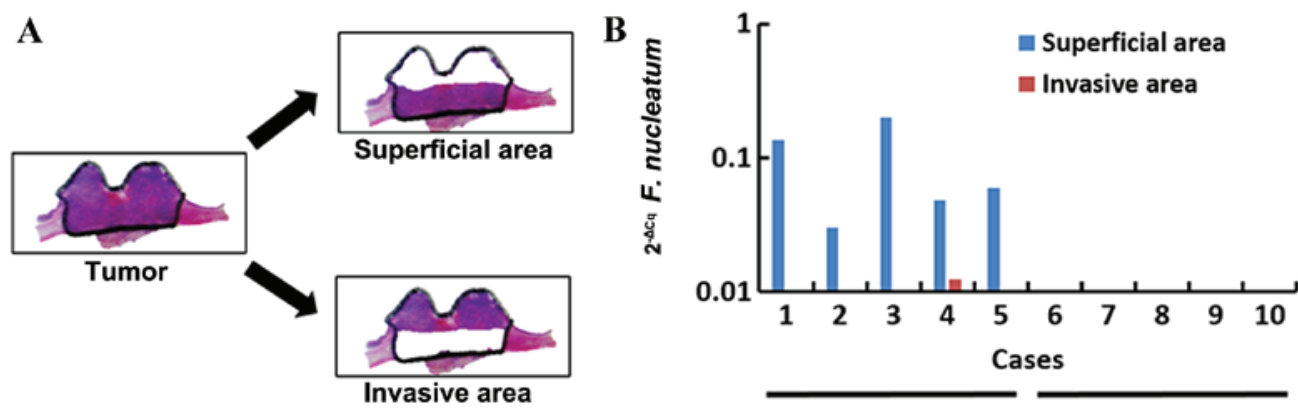

$(+)$

F. nucleatum

Figure 3. F. nucleatum DNA in superficial and invasive tissue areas. (A) Macrodissection of the superficial and invasive areas in tissues that were positive (cases 1-5) and negative (cases 6-10) for F. nucleatum. (B) F. nucleatum DNA was observed at high levels in superficial areas (range $30.1 \times 10^{-3}-200.3 \times 10^{-3}$ ) and at low levels in invasive areas $\left(0-12.4 \times 10^{-3} ; \mathrm{P}=0.02\right) . F$., Fusobacterium.

and their adjacent non-tumor tissues. Among all cancer cases that were positive for $F$. nucleatum, the level of $F$. nucleatum DNA content in esophageal and colorectal cancer ranged from $2.68 \times 10^{-3}$ to $365.2 \times 10^{-3}$ (median, $101.3 \times 10^{-3}$ ) and from $2.10 \times 10^{-3}$ to $166.7 \times 10^{-3}$ (median, $5.08 \times 10^{-3}$ ), respectively.

Heterogeneity of $F$. nucleatum in esophageal cancer tissue. To evaluate the heterogeneity of the F. nucleatum DNA within tumor tissues, the $F$. nucleatum DNA in the superficial and invasive areas of the 5 esophageal cancer tissues that were positive for $F$. nucleatum were evaluated (Fig. 3A). High levels of Fusobacterium nucleatum DNA was observed in superficial areas, but low levels were observed in invasive areas. In the superficial areas, the quantity of $F$. nucleatum DNA ranged from $30.1 \times 10^{-3}$ to $200.3 \times 10^{-3}$, whereas in invasive areas it was $12.4 \times 10^{-3}$ at its highest $(\mathrm{P}=0.02$; Fig. $3 \mathrm{~B})$. Therefore, the F. nucleatum DNA may distribute heterogeneously within a single tumor.

\section{Discussion}

F. nucleatum has received increased recognition as an opportunistic pathogen in periodontal diseases, but also in human cancer. As the microbiome has a number of important effects 
on the functions of the human body, the clinical significance of the discovery of $F$. nucleatum cannot be overemphasized. To the best of our knowledge, the present study has reported the first detection of $F$. nucleatum DNA in esophageal, gastric and liver cancer tissues. The present study has demonstrated that the qPCR assay may reliably detect $F$. nucleatum DNA from oral swabs, as $F$. nucleatum is among the most prevalent species in the oral cavity $(1,2,24)$. The association between cycle threshold and input DNA in the qPCR assay of F. nucleatum was linear $\left(r^{2}>0.99\right)$. Furthermore, the FFPE and frozen tissues prepared from the same esophageal tumor yielded a similar level of detection accuracy. Typically, the fixation process chemically alters the nucleic acids in a sample by inducing covalent DNA cross-linking and fragmentation. These alterations may reduce the efficacy of analysis using PCR and DNA sequencing methods $(25,26)$. In the present study, the results of the FFPE and frozen tissues were concordant, which suggested that in the two types of tissue preparations, qPCR accurately detected $F$. nucleatum DNA in gastroenterological cancer tissues.

F. nucleatum DNA was successfully detected in gastrointestinal tract cancer tissues (esophageal, gastric and colorectal cancer), but $F$. nucleatum was not detected in pancreatic and liver cancer tissues. In previous studies, the detection rates of $F$. nucleatum were $13-82 \%$ in colorectal cancer $(15,16,19,21)$ and $8.8 \%$ in pancreatic cancer $(20)$. These previous studies are concordant with the data from the present study that uses colorectal cancer tissues, but these results contradict the pancreatic cancer results in the current study. Although F. nucleatum is part of the normal flora of the oropharyngeal and gastrointestinal tracts, $F$. nucleatum also expresses FadA, a bacterial cell surface adhesion protein that activates the WNT signaling pathway in colorectal cancer cells, and consequently promotes tumor growth (14). Therefore, it may reasonably be expected that the detection rate of $F$. nucleatum is higher in gastroenterological cancer compared with liver and pancreatic cancer. However, the presence of $F$. nucleatum in esophageal and gastric cancer tissues remains to be investigated.

In addition, the $F$. nucleatum expression levels in superficial and invasive areas of esophageal cancer tissues were compared, and an increased level was observed in superficial areas. This result suggested that $F$. nucleatum may not be able to infiltrate into the invasive area and may only contribute to the tumor growth through the side of the gastrointestinal tract. As the differential distribution of $F$. nucleatum has not been previously reported, the low level of $F$.nucleatum in invasive areas remains to be fully elucidated. The involvement of $F$. nucleatum in tumor growth requires further investigation.

In conclusion, $F$. nucleatum DNA was detected in esophageal, gastric and colorectal cancer, but not in pancreatic and liver cancer. This suggested that $F$. nucleatum may be associated with the progression of gastroenterological tract cancer, but not the progression of pancreatic and liver cancer.

\section{Acknowledgements}

The present study was supported in part by 27 th $\mathrm{SGH}$ Foundation.

\section{References}

1. Griffen AL, Beall CJ, Campbell JH, Firestone ND, Kumar PS, Yang ZK, Podar M and Leys EJ: Distinct and complex bacterial profiles in human periodontitis and health revealed by $16 \mathrm{~S}$ pyrosequencing. ISME J 6: 1176-1185, 2012.

2. Loozen G, Ozcelik O, Boon N, De Mol A, Schoen C, Quirynen M and Teughels $\mathrm{W}$ : Inter-bacterial correlations in subgingival biofilms: A large-scale survey. J Clin Periodontol 41: 1-10, 2014.

3. Feng X, Zhang L, Xu L, Meng H, Lu R, Chen Z, Shi D and Wang X: Detection of eight periodontal microorganisms and distribution of Porphyromonas gingivalis fim A genotypes in Chinese patients with aggressive periodontitis. J Periodontol 85: 150-159, 2014.

4. Liu P, Liu Y, Wang J, Guo Y, Zhang Y and Xiao S: Detection of fusobacterium nucleatum and fadA adhesin gene in patients with orthodontic gingivitis and non-orthodontic periodontal inflammation. PLoS One 9: e85280, 2014.

5. Ohkusa T, Okayasu I, Ogihara T, Morita K, Ogawa M and Sato N: Induction of experimental ulcerative colitis by Fusobacterium varium isolated from colonic mucosa of patients with ulcerative colitis. Gut 52: 79-83, 2003

6. Minami M, Ando T, Okamoto A, Sasaki N, Ohkura T, Torii K, Hasegawa T, Ohta M and Goto H: Seroprevalence of Fusobacterium varium in ulcerative colitis patients in Japan. FEMS Immunol Med Microbiol 56: 67-72, 2009.

7. Tahara T, Shibata T, Kawamura T, Okubo M, Ichikawa Y, Sumi K, Miyata M, Ishizuka T, Nakamura M, Nagasaka M, et al: Fusobacterium detected in colonic biopsy and clinicopathological features of ulcerative colitis in Japan. Dig Dis Sci 60: 205-210, 2015.

8. Strauss J, Kaplan GG, Beck PL, Rioux K, Panaccione R, Devinney R, Lynch T and Allen-Vercoe E: Invasive potential of gut mucosa-derived Fusobacterium nucleatum positively correlates with IBD status of the host. Inflamm Bowel Dis 17: 1971-1978, 2011.

9. Song YG, Shim SG, Kim KM, Lee DH, Kim DS, Choi SH, Song JY, Kang HL, Baik SC, Lee WK, et al: Profiling of the bacteria responsible for pyogenic liver abscess by $16 \mathrm{~S}$ rRNA gene pyrosequencing. J Microbiol 52: 504-509, 2014.

10. Yoneda M, Kato S, Mawatari H, Kirikoshi H, Imajo K, Fujita K, Endo H, Takahashi H, Inamori M, Kobayashi N, et al: Liver abscess caused by periodontal bacterial infection with Fusobacterium necrophorum. Hepatol Res 41: 194-196, 2011.

11. Bohrer JC, Kamemoto LE, Almeida PG and Ogasawara KK: Acute chorioamnionitis at term caused by the oral pathogen Fusobacterium nucleatum. Hawaii J Med Public Health 71: 280-281, 2012.

12. Castellarin M, Warren RL, Freeman JD, Dreolini L, Krzywinski M, Strauss J, Barnes R, Watson P, Allen-Vercoe E, Moore RA, et al: Fusobacterium nucleatum infection is prevalent in human colorectal carcinoma. Genome Res 22: 299-306, 2012.

13. Kostic AD, Gevers D, Pedamallu CS, Michaud M, Duke F, Earl AM, Ojesina AI, Jung J, Bass AJ, Tabernero J, et al: Genomic analysis identifies association of Fusobacterium with colorectal carcinoma. Genome Res 22: 292-298, 2012.

14. Rubinstein MR, Wang X, Liu W, Hao Y, Cai G and Han YW: Fusobacterium nucleatum promotes colorectal carcinogenesis by modulating E-cadherin/beta-catenin signaling via its FadA adhesin. Cell Host Microbe 14: 195-206, 2013.

15. Tahara T, Yamamoto E, Suzuki H, Maruyama R, Chung W, Garriga J, Jelinek J, Yamano HO, Sugai T, An B, et al: Fusobacterium in colonic flora and molecular features of colorectal carcinoma. Cancer Res 74: 1311-1318, 2014.

16. Mima K, Sukawa Y, Nishihara R, Qian ZR, Yamauchi M, Inamura K, Kim SA, Masuda A, Nowak JA, Nosho K, et al: Fusobacterium nucleatum and $\mathrm{T}$ cells in colorectal carcinoma. JAMA Oncol 1: 653-661, 2015.

17. Flanagan L, Schmid J, Ebert M, Soucek P, Kunicka T, Liska V, Bruha J, Neary P, Dezeeuw N, Tommasino M, et al: Fusobacterium nucleatum associates with stages of colorectal neoplasia development, colorectal cancer and disease outcome. Eur J Clin Microbiol Infect Dis 33: 1381-1390, 2014.

18. Mima K, Nishihara R, Qian ZR, Cao Y, Sukawa Y, Nowak JA, Yang J, Dou R, Masugi Y, Song M, et al: Fusobacterium nucleatum in colorectal carcinoma tissue and patient prognosis. Gut 65: 1973-1980, 2016.

19. Ito M, Kanno S, Nosho K, Sukawa Y, Mitsuhashi K, Kurihara H, Igarashi $\mathrm{H}$, Takahashi $\mathrm{T}$, Tachibana $\mathrm{M}$, Takahashi $\mathrm{H}$, et al: Association of Fusobacterium nucleatum with clinical and molecular features in colorectal serrated pathway. Int J Cancer 137: 1258-1268, 2015. 
20. Mitsuhashi K, Nosho K, Sukawa Y, Matsunaga Y, Ito M, Kurihara H, Kanno S, Igarashi H, Naito T, Adachi Y, et al: Association of Fusobacterium species in pancreatic cancer tissues with molecular features and prognosis. Oncotarget 6: 7209-7220, 2015.

21. Viljoen KS, Dakshinamurthy A, Goldberg P and Blackburn JM: Quantitative profiling of colorectal cancer-associated bacteria reveals associations between fusobacterium spp., enterotoxigenic Bacteroides fragilis (ETBF) and clinicopathological features of colorectal cancer. PLoS One 10: e0119462, 2015.

22. Compton CC, Byrd DR, Garcia-Aguilar J, Kurtzman SH, Olawaiye A and Washington MK (eds): The AJCC cancer staging atlas, 2nd edition. Springer, New York, NY, 2012.
23. Livak KJ and Schmittgen TD: Analysis of relative gene expression data using real-time quantitative PCR and the 2- $\Delta \Delta \mathrm{CT}$ method. Methods 25: 402-408, 2001

24. Field CA, Gidley MD, Preshaw PM and Jakubovics N: Investigation and quantification of key periodontal pathogens in patients with type 2 diabetes. J Periodontal Res 47: 470-478, 2012.

25. Do H and Dobrovic A: Dramatic reduction of sequence artefacts from DNA isolated from formalin-fixed cancer biopsies by treatment with uracil-DNA glycosylase. Oncotarget 3: 546-558, 2012.

26. Sah S, Chen L, Houghton J, Kemppainen J, Marko AC, Zeigler R and Latham GJ: Functional DNA quantification guides accurate next-generation sequencing mutation detection in formalin-fixed, paraffin-embedded tumor biopsies. Genome Med 5: 77, 2013. 